'Departamento de Anatomía Humana. Escuela de Medicina. Universidad de Cádiz. Cádiz, España.

${ }^{2}$ Escuela de Medicina. Universidad de Málaga. Málaga, España. ${ }^{3}$ Departamento de Medicina. Escuela de Medicina. Universidad de Cádiz. Cádiz, España.

${ }^{4}$ División de Urología. Hospita Universitario Puerta del Mar. Cádiz, España. ${ }^{5}$ School of Sport Sciences. Universidad de Cádiz. Cádiz, España.

${ }^{6}$ División de Ginecología. Hospital Universitario de Jerez. Cádiz, España. aphD.

Recibido el 8 de agosto de 2013 aceptado el 17 de junio de 2014

Correspondencia a: Francisco Javier Ordoñez MD PhD Departamento de Anatomía Humana. Escuela de Medicina. Universidad de Cádiz. Pza. Fragela s/n 11003 Cádiz, España

Phone. +34 617682043

Fax. +34956015254

franciscojavier.ordonez@uca.es

\section{Asociación entre obesidad abdominal y daño oxidativo seminal en pacientes con síndrome metabólico}

\author{
IGNACIO ROSETY ${ }^{1, a}$, SONIA ELOSEGUI ${ }^{2}$, MARÍA T. PERY ${ }^{3}$, \\ GABRIEL FORNIELES ${ }^{3}$, JESÚS M. ROSETY ${ }^{4}$, \\ ANTONIO J. DÍAZ ${ }^{3, a}$, ANTONIA RODRÍGUEZ-PAREJA ${ }^{5}$, \\ FRANCISCO BRENES ${ }^{3}$, MANUEL ROSETY-RODRÍGUEZ ${ }^{3}$, \\ FRANCISCO J. ORDONEZ ${ }^{1}$, MIGUEL A. ROSETY, ${ }^{6, a}$
}

\section{Association between abdominal obesity and seminal oxidative damage in adults with metabolic syndrome}

Background: Abdominal obesity, metabolic syndrome (MS) and oxidative stress may impair seminal quality leading to derangements in fertility. Aim: To identify an association between abdominal obesity and markers of seminal oxidative damage in adults with MS. Material and Methods: Seventy males aged 25 to 40 years, with MS according to ATP-III criteria volunteered for this cross-sectional study. The control group included 70 healthy and normal weight adults. Semen analysis included volume, sperm concentration, motility and normal morphologic features. Body mass index (BMI) and waist circumference (WC) were measured, fat mass was determined by bioelectrical impedance. Results: Sperm concentration and the percentage of sperms with normal motility and morphology were significantly lower in adults with MS, when compared to their healthy normal weight counterparts. Seminal levels of malondialdehyde and 8-hydroxy-2'-deoxyguanosine were significantly higher among participants with MS. Significant correlations were found between WC and seminal markers of oxidative stress. Conclusions: Individuals with MS had an impaired seminal quality that may be explained, at least in part, by increased seminal oxidative damage.

(Rev Med Chile 2014; 142: 732-737)

Key words: Metabolic syndrome X; Oxidative stress; Semen.
E 1 síndrome metabólico está recibiendo cada vez mayor atención en la literatura especializada debido a su creciente prevalencia ${ }^{1}$ así como a los altos costes sanitarios que genera ${ }^{2}$.

Aunque en la actualidad sigue existiendo una controversia respecto a qué clasificación diagnóstica utilizar a nivel asistencial para prevenir futuros eventos cardiovasculares ${ }^{3}$, todas coinciden gran interés a la obesidad abdominal, presente como perímetro de la cintura ${ }^{4}$.

De igual modo, existe un amplio consenso al aceptar la importancia del daño oxidativo en la fisiopatología del síndrome metabólico ${ }^{5}$. De hecho, éste ha sido propuesto como diana terapéutica sobre la que actuar mediante fármacos como estatinas ${ }^{6}$.

Más recientemente, diversos estudios han puesto de manifiesto que los pacientes con síndrome metabólico podrían presentar problemas de fertilidad que sería atribuible a la deficiente calidad y concentración de su semen ${ }^{7}$. También se acepta que el daño oxidativo reduce signifi- 
cativamente la cantidad y calidad (motilidad; morfología) del espermatozoide en pacientes infértiles ${ }^{8}$. Precisamente, en los últimos años, marcadores de daño oxidativo de lípidos como malondialdehido (MDA) han sido ampliamente utilizados en la literatura ${ }^{8,9}$. Paralelamente, estudiar la oxidación del ADN resulta de especial interés ya que éste podría impactar no sólo en la infertilidad masculina sino también en la salud de la descendencia ${ }^{10}$.

Por consiguiente, nos planteamos como hipótesis de trabajo que los pacientes con síndrome metabólico presentan un mayor daño oxidativo seminal que podría afectar la calidad de su semen. Paralelamente pretendemos identificar correlaciones estadísticamente significativas entre obesidad abdominal y marcadores de daño oxidativo a nivel seminal.

\section{Material y Métodos}

\section{Participantes}

En nuestro estudio transversal participaron voluntariamente 70 adultos jóvenes (25-40 años) con diagnóstico de síndrome metabólico, definido según los criterios NCEP-ATP-III ${ }^{11}$, procedentes de Centros de Atención Primaria. El grupo control estaba formado por 70 adultos jóvenes con normopeso sin diagnóstico de síndrome metabólico. Como criterios de exclusión en ambos grupos se consideraron: 1) Varicocele; 2) Reversión de vasectomía; 3 ) infección aguda/crónica de tracto genitourinario; 4) Participación en programas de actividad física o regímenes dietéticos en los últimos 6 meses; 5) Hábitos tóxicos (alcohol y tabaco); 6) Consumo suplementos nutricionales antioxidantes; 7) Ocupación profesional como conductor.

\section{Análisis de la cantidad y calidad de espermatozoides}

Las muestras de semen se obtuvieron mediante masturbación tras 3 días de abstinencia, coleccionándose en tubos estériles. En un plazo máximo de 30 min se realizó el análisis seminal incluyendo volumen seminal (ml), concentración de espermatozoides (\%), motilidad (\%) y morfología normal (\%). Dichos parámetros se evaluaron de acuerdo con las recomendaciones previstas por al Organización Mundial de la Salud ${ }^{12}$. De manera más detallada merece ser puntualizado que la concentración espermática se obtuvo mediante el uso de un hemocitómetro (Hauser Scientific Inc., Horsham, PA). El porcentaje de espermatozoides móviles se determinó mediante el método CASA (CASA system, Microptic S.L., España).

\section{Defensas antioxidantes y marcadores de daño oxidativo seminales}

Las muestras de plasma seminal se obtuvieron tras un proceso de centrifugado a $3.500 \mathrm{rpm} \mathrm{du}-$ rante $15 \mathrm{~min}$, coleccionándose el sobrenadante en tubos de Eppendorf criogénicos $(3 \mathrm{ml})$ y conservándose $\mathrm{a}-80^{\circ} \mathrm{C}$ hasta su análisis.

Los niveles de malondialdehido (MDA), como marcador de la oxidación de lípidos, se realizó mediante el método de Yagi ${ }^{13}$ utilizando técnicas espectrofotométricas. Los resultados se expresaron como nmol MDA $/ 10^{7}$ células.

El porcentaje de espermatozoides que presentaba 8 -hidroxideoxiguanosina ( $8 \mathrm{OHdG}$ ), principal biomarcador de la oxidación del ADN, se determinó mediante el método de Kao et al. ${ }^{14}$, utilizando anticuerpos específicos marcados con fluoresceína-5-isotiocianato (FITC) disponible en kits comerciales OxyDNA Test (Biotrin International Ltd, Dublin, Irlanda).

\section{Valoración antropométrica}

El porcentajes de masa grasa se determinó mediante impedanciometría bioeléctrica utilizando un modelo segmental multifrecuencia (Tanita MC-180MA) por ser aquellos que se utilizan habitualmente a nivel experimental ${ }^{15}$. Los participantes fueron invitados a orinar antes de someterse a esta prueba. También se les recomendó no ingerir bebidas estimulantes así como la no participación en actividad física intensa.

El perímetro de cintura se determinó mediante cinta antropométrica siguiendo las indicaciones de la ISAK (International Society for the Advancement of Kinanthropometry $)^{16}$.

\section{Aspectos éticos}

Tras una sesión informativa los participantes entregaron debidamente firmada la hoja de consentimiento. Se atendieron las recomendaciones previstas en la Declaración de Helsinki (versión 2002) de la Asociación Médica Mundial. Este protocolo fue aprobado por un Comité de Ética Institucional Universitario. 
Obesidad abdominal y daño oxidativo seminal en síndrome metabólico - I. Rosety et al

Tabla 1. Características antropométricas, bioquímicas y clínicas de jóvenes adultos con síndrome metabólico $(n=70)$ y controles sanos con normopeso $(n=70)$

\begin{tabular}{|c|c|c|c|}
\hline & Síndrome metabólico & Controles normopeso & $\mathbf{p}$ \\
\hline Edad (años) & $34,8 \pm 5,1$ & $35,4 \pm 4,2$ & $>0,05$ \\
\hline Masa grasa (\%) & $33,5 \pm 3,7$ & $25,7 \pm 3,3$ & $<0,05$ \\
\hline IMC & $32,0 \pm 1,7$ & $23,6 \pm 1,5$ & $<0,05$ \\
\hline$P C(\mathrm{~cm})$ & $106,4 \pm 5,7$ & $86,2 \pm 4,9$ & $<0,05$ \\
\hline $\mathrm{c}-\mathrm{HDL}(\mathrm{mg} / \mathrm{dl})$ & $39,7 \pm 2,5$ & $61,3 \pm 2,2$ & $<0,05$ \\
\hline Trigliceridos (mg/dl) & $159,3 \pm 13,0$ & $126,4 \pm 12,6$ & $<0,05$ \\
\hline Glucemia (mg/dl) & $107,6 \pm 6,5$ & $98,9 \pm 6,1$ & $<0,05$ \\
\hline TAS (mm Hg) & $124,8 \pm 10,9$ & $110,7 \pm 10,1$ & $<0,05$ \\
\hline $\mathrm{TAD}(\mathrm{mm} \mathrm{Hg})$ & $87,0 \pm 6,2$ & $76,4 \pm 5,5$ & $<0,05$ \\
\hline
\end{tabular}

Note: Resultados expresados como media \pm sd. IMC: Índice cintura/cadera; PC: Perímetro de la cintura. c-HDL: Colesterol-HDL. TAS: Tensión arterial sistólica; TAD: Tensión arterial diastólica.

\section{Análisis estadístico}

Los resultados se expresarán como media \pm sd. La distribución de normalidad se realizará mediante el test de Shapiro-Wilk habida cuenta de lo limitada de la población objeto de estudio. La comparación de medias se realizará mediante análisis de la varianza (ANOVA) de una sola vía, con la aplicación post-hoc del test corrector de Bonferroni. El nivel de significación se situó en un valor de $\mathrm{p}<0,05$.

\section{Resultados}

Las características antropométricas, bioquímicas y clínicas de los grupos de intervención y control se resumen en la Tabla 1.

La calidad seminal entre los pacientes con síndrome metabólico fue peor que la de controles sanos con normopeso. En este sentido, la concentración $(\mathrm{p}=0,018)$, y los porcentajes de espermatozoides móviles $(\mathrm{p}=0,026)$ y con morfología normal $(p=0,041)$ fueron significativamente menores entre aquellos adultos que cumplían los criterios diagnósticos de síndrome metabólico. Todos estos resultados se detallan en la Tabla 2.

También se observó un mayor daño oxidativo seminal entre los pacientes con síndrome metabólico. De manera más detallada, la lipoperoxidación, expresada como niveles de MDA fue significativamente mayor entre los adultos con síndrome metabólico $(\mathrm{p}=0,029)$. En cuanto al daño oxidativo sobre el ADN, el porcentaje de espermatozoides con presencia de $8 \mathrm{OHdG}$ también fue significativamente mayor entre pacientes con síndrome metabólico cuando se compara con controles sanos normopeso $(\mathrm{p}=0,033)$. Dichos resultados se enumeran en la Tabla 2.

Finalmente, se han puesto de manifiesto correlaciones estadísticamente significativas entre índices de distribución abdominal de masa grasa (PC) y los parámetros seminales ensayados. Por un lado, se observaron correlaciones significativas, aunque de naturaleza negativa, entre PC y concentración $(\mathrm{r}=-0,33 ; \mathrm{p}=0,0284)$ motilidad $(\mathrm{r}=-0,30 ; \mathrm{p}=0,0311)$ y normal morfología $(r=-0,29 ; p=0,0342)$ espermáticas. Por otra parte, se ha evidenciado la existencia de asociaciones significativas entre PC y marcadores de daño oxidativo seminal como MDA $(r=0,46 ; p=0,0170)$ y 8 OHdG $(r=0,39 ; p=0,0312)$.

\section{Discusión}

Nuestros resultados han confirmado que las personas con síndrome metabólico presentan una calidad seminal menor que la de pacientes sanos con normopeso.

Recientes estudios han demostrado que la obesidad abdominal o central está asociada con menores concentraciones de espermatozoides y 
Tabla 2. Evaluación estándar del fluido seminal y marcadores de daño oxidativo en adultos con síndrome metabólico y controles sanos con normopeso

\begin{tabular}{|lccc|}
\hline & Síndrome metabólico & Controles normopeso & p valor \\
Volumen & $2,9 \pm 0,9$ & $3,2 \pm 0,8$ & 0,067 \\
Concentración & $60,3 \pm 6,4$ & $69,7 \pm 6,1$ & 0,018 \\
Movilidad & $58,1 \pm 6,0$ & $67,7 \pm 5,6$ & 0,026 \\
Morfología & $13,6 \pm 4,1$ & $15,1 \pm 4,4$ & 0,046 \\
Malondialdehido & $30,0 \pm 5,4$ & $19,1 \pm 5,1$ & 0,029 \\
8-OHdG & $28,2 \pm 4,7$ & $17,6 \pm 4,9$ & 0,033 \\
\hline
\end{tabular}

Nota: Resultados expresados como media \pm sd. Volumen expresado en ml. Concentración expresada como 10\%/ml; Movilidad y morfología expresados como porcentaje. MDA: Malondialdehido expresado como nmol MDA $10^{7}$ células 8-OHdG: 8-hidroxideoxiguanosina expresado como porcentaje.

porcentajes de movilidad ${ }^{17}$. Este hecho es de especial interés ya que la obesidad abdominal es uno de los criterios que mayor atención ha recibido en las diferentes clasificaciones diagnósticas existentes para síndrome metabólico. Entre las razones que podrían explicar dicha asociación destaca que el exceso de masa grasa podría alterar los niveles de hormonas reproductoras, reduciendo los niveles de testosterona e incrementando los de estradiol ${ }^{18}$. Más recientemente, $\mathrm{McD}$ onald et al. ${ }^{19}$ han demostrado que la disrupción endocrina que presentan pacientes con sobrepeso u obesidad impacta negativamente en su calidad seminal. Sin embargo, al revisar la literatura no existe un consenso total sobre este asunto, ya que otros autores no han encontrado una correlación estadísticamente significativa entre dichas variables ${ }^{20}$. Por consiguiente, futuros estudios en esta línea de trabajo son aún necesarios

Por otra parte, la mala calidad seminal que hemos observado en pacientes con síndrome metabólico podría explicarse, al menos en parte, por los mayores niveles de daño oxidativo observados en el plasma seminal de los participantes en nuestro estudio.

Aunque en la actualidad aún existe un intenso debate sobre los criterios diagnósticos del síndrome metabólico, parece existir un mayor acuerdo en la implicación del daño oxidativo en su fisiopatología debido a una excesiva producción de especies reactivas de oxígeno (ROS) ${ }^{21}$.

De hecho, el estrés oxidativo es ampliamente reconocido como una de las principales causas de infertilidad masculina ${ }^{8}$. De manera más detallada, niveles elevados de marcadores de daño oxidativo del ADN se asocia a malformaciones espermáticas $^{22}$. Asimismo, la acción de las ROS sobre fosfolípidos poliinsaturados de membrana espermática se ha asociado a malformaciones y afectación de la movilidad ${ }^{23}$. En esta misma línea, una revisión sistemática sobre la utilidad de la suplementación oral con antioxidantes (vitaminas $\mathrm{C}$ y E, Selenio, Zinc, etc.) concluyó que 14 de un total de 17 ensayos seleccionados encontraron mejoras de la calidad del semen, aumentando la frecuencia de embarazos y de nacimientos vivos ${ }^{24}$. Sin embargo, se debe tener presente que el abuso de vitaminas antioxidantes, especialmente de naturaleza liposoluble como la vitamina E, puede asociarse a efectos secundarios al convertirse ella misma en un radical $\alpha$-tocoferoxil ${ }^{25}$. Estudios previos han puesto de manifiesto que el ejercicio físico de tipo aeróbico podría reducir el daño oxidativo en pacientes con síndrome metabólico mediante un incremento significativo de los niveles de defensas antioxidantes $^{23,26}$.

Uno de los aspectos más novedosos de nuestro trabajo fue la identificación de correlaciones entre obesidad abdominal y marcadores de calidad seminal en pacientes con síndrome metabólico. De manera más detallada la mayor fuerza de asociación, aunque de naturaleza negativa, se estableció entre el perímetro de la cintura y la concentración de espermatozoides y el porcentaje de movilidad de los mismos. Resultados similares fueron publicados anteriormente por Hammiche et al. $^{17}$ confirmando el impacto negativo de la obesidad abdominal en la calidad seminal. Por 
el contrario, Eskandar et al. ${ }^{20}$ no encontraron correlaciones significativas entre el índice de masa corporal (IMC) y marcadores de calidad seminal. Sin embargo estos mismos autores no estudiaron ningún índice de distribución de masa grasa abdominal (p. ej. perímetro de la cintura; perímetro de la cadera; índice cintura/cadera) para confirmar sus resultados.

Paralelamente, se ha puesto de manifiesto una correlación significativa entre el perímetro de la cintura y los niveles de MDA en plasma seminal, principal marcador de lipoperoxidación. Este hecho podría explicarse, al menos en parte, por la abundancia en las membranas de los espermatozoides de ácidos grasos poli-insaturados (PUFAs) que son altamente susceptibles a las especies reactivas de oxígeno $(\mathrm{ROS})^{20}$.

Coincidiendo con estudios previos ${ }^{27,28}$, nuestros resultados sugieren que la degradación de las membranas espermáticas mediante la lipoperoxidación podría justificar las alteraciones observadas en la calidad del semen, especialmente en lo que respecta a la movilidad y morfología de los espermatozoides.

El actual estudio presenta algunas limitaciones. No se han determinado los niveles de hormonas reproductoras como testosterona, estradiol, FSH o LH entre otras a pesar de la importancia del la obesidad abdominal en sus niveles plasmáticos ${ }^{18,19}$. Tampoco se han incluido marcadores plasmáticos de defensas antioxidantes o daño oxidativo si bien recientes estudios han confirmado que existe una fuerte correlación con los parámetros obtenidos a nivel seminal ${ }^{29}$. El tamaño muestral también podría limitar la generalización de los resultados derivados de este estudio piloto.

Por otra parte, destacamos como fortalezas que a diferencia de la mayoría de estudios previos que se han desarrollado en centros de reproducción asistida sobre cohortes de varones subfértiles o infértiles, lo que podría suponer un sesgo importante por la presencia de factores de confusión, el nuestro se ha desarrollado en la comunidad. Asimismo, otros estudios han utilizado parámetros sobre estilo de vida y datos antropométricos facilitados por los propios participantes que podrían sub/sobreestimar algunos de ellos ${ }^{30}$. Por último, haber utilizado parámetros antropométricos convencionales en vez de pruebas de imagen facilita la reproducibilidad y aplicabilidad de este estudio en cualquier entorno clínico.
Se concluye que los pacientes con síndrome metabólico presentan una menor calidad seminal que podría atribuirse entre otras razones a la obesidad abdominal así como a un mayor daño oxidativo. También se ha observado una correlación significativa entre la obesidad abdominal y el daño oxidativo seminal que podría facilitar el seguimiento clínico de estos pacientes. Futuros estudios que identifiquen estrategias para mejorar la calidad del semen en este grupo poblacional son aún necesarios.

\section{Referencias}

1. Valenzuela AA, Maíz A, Margozzini P, Ferreccio C, Rigotti A, Olea R, et al. [Prevalence of metabolic syndrome among Chilean adults]. Rev Med Chile 2010; 138: 70714.

2. Scholze J, Alegría E, Ferri C, Langham S, Stevens W, Jeffries D, et al. Epidemiological and economic burden of metabolic syndrome and its consequences in patients with hypertension in Germany, Spain and Italy; a prevalence-based model. BMC Public Health 2010; 10: 529.

3. Sarmiento Méndez LM, Roca-Cusachs Coll A, Arroyo Díaz JA, Benet Gustà MT, Solé Villa MJ, Franco Peral M. Comparison of the definitions of the metabolic syndrome according to ATP III and IDF. Rev Clin Esp 2008; 208: 333-8.

4. Després JP, Lemieux I, Bergeron J, Pibarot P, Mathieu $\mathrm{P}$, Larose $\mathrm{E}$, et al. Abdominal obesity and the metabolic syndrome: contribution to global cardiometabolic risk. Arterioscler Thromb Vasc Biol 2008; 28: 1039-49.

5. Holvoet P, Lee DH, Steffes M, Gross M, Jacobs DR Jr. Association between circulating oxidized low-density lipoprotein and incidence of the metabolic syndrome. Jama 2008; 299: 2287-93.

6. Lazich I, Sarafidis P, de Guzmán E, Patel A, Oliva R, Bakris G. Effects of combining simvastatin with rosiglitazone on inflammation, oxidant stress and ambulatory blood pressure in patients with the metabolic syndrome: the SIROCO study. Diabetes Obes Metab 2012; 14: 1816.

7. Michalakis K, Mintziori G, Kaprara A, Tarlatzis BC, Goulis DG. The complex interaction between obesity, metabolic syndrome and reproductive axis: a narrative review. Metabolism 2013; 62: 457-78.

8. Benedetti S, Tagliamonte MC, Catalani S, Primiterra M, Canestrari F, De Stefani S, et al. Differences in blood and semen oxidative status in fertile and infertile men, and 
their relationship with sperm quality. Reprod Biomed Online 2012; 25; 300-6.

9 Hajizadeh Maleki B, Tartibian B, Eghbali M, Asri-Rezaei S. Comparison of seminal oxidants and antioxidants in subjects with different levels of physical fitness. Andrology 2013; 1: 607-14.

10. Aitken RJ, De Iuliis GN, Finnie JM, Hedges A, McLachlan RI. Analysis of the relationships between oxidative stress, DNA damage and sperm vitality in a patient population: development of diagnostic criteria. Hum Reprod 2010; 25: 2415-26.

11. Grundy SM, Cleeman JI, Daniels SR, Donato KA, Eckel $\mathrm{RH}$, Franklin BA, et al. Diagnosis and management of the metabolic syndrome: an American Heart Association/National Heart, Lung, and Blood Institute scientific statement. Circulation 2005; 112: 2735-52.

12. World Health Organization. Laboratory manual for the examination of human semen and sperm-cervical mucus interaction. Cambridge: Cambridge University Press; 1999.

13. Yagi K. A simple fluorometric assay for lipoperoxide in blood plasma. Biochem Med 1976; 5: 212-6.

14. Kao SH, Chao HT, Chen HW, Hwang TI, Liao TL, Wei YH. Increase of oxidative stress in human sperm with lower motility. Fertil Steril 2008; 89: 1183-90.

15. Bosy-Westphal A, Schautz B, Later W, Kehayias JJ, Gallagher D, Müller MJ. What makes a BIA equation unique? Validity of eight-electrode multifrequency BIA to estimate body composition in a healthy adult population. Eur J Clin Nutr 2013; 67: S14-21.

16. Marfell-Jones, M, Olds T, Stewart A, Carter L. International Standards for Anthropometric Assessment. Potchefstroom (South Africa): North-West University; 2006.

17. Hammiche F, Laven JS, Twigt JM, Boellaard WP, Steegers EA, Steegers-Theunissen RP. Body mass index and central adiposity are associated with sperm quality in men of subfertile couples. Hum Reprod 2012; 27: 2365-72.

18. Al-Ali B, Gutschi T, Pummer K, Zigeuner R, BrookmanMay S, Wieland WF, et al. Body mass index has no impact on sperm quality but on reproductive hormones levels. Andrologia. 2012 Nov 24. doi: 10.1111/and.12051.

19. Macdonald AA, Stewart AW, Farquhar CM. Body mass index in relation to semen quality and reproductive hormones in New Zealand men: a cross-sectional study in fertility clinics. Hum Reprod. 2013 Oct 15. [Epub ahead of print].
20. Eskandar M, Al-Asmari M, Babu Chaduvula S, Al-Shahrani M, Al-Sunaidi M, Almushait M, et al. Impact of male obesity on semen quality and serum sex hormones. Adv Urol. 2012. doi: 10.1155/2012/407601.

21. Kotani K, Sakane N. C-reactive protein and reactive oxygen metabolites in subjects with metabolic syndrome. J Int Med Res 2012; 40: 1074-81.

22. Guz J, Gackowski D, Foksinski M, Rozalski R, Zarakowska E, Siomek A, et al. Comparison of oxidative stress/DNA damage in semen and blood of fertile and infertile men. PLoS One 2013; 8 (7): e68490.

23. Atig F, Raffa M, Habib BA, Kerkeni A, Saad A, Ajina M. Impact of seminal trace element and glutathione levels on semen quality of Tunisian infertile men. BMC Urol 2012; $12: 6$.

24. Ross C, Morriss A, Khairy M, Khalaf Y, Braude P, Coomarasamy A, et al. A systematic review of the effect of oral antioxidants on male infertility. Reprod Biomed Online 2010; 20: 711-23.

25. No authors listed. Vitamins and supplements: 10 dangers that may surprise you. Consum Rep 2012; 77: 1823.

26. Rosety-Rodríguez M, Díaz-Ordoñez A, Rosety I, Fornieles G, Camacho-Molina A, García N, et al. [Aerobic training improves antioxidant defense system in women with metabolic syndrome]. Medicina (B Aires) 2012; 72: 15-8.

27. Eleutério-Silva MA, da Fonseca LJ, Velloso EP, da Silva Guedes G, Sampaio WO, da Silva WF, et al. Short-term cardiovascular physical programme ameliorates arterial stiffness and decreases oxidative stress in women with metabolic syndrome. J Rehabil Med 2013; 45: 572-9.

28. Ben Abdallah F, Dammak I, Attia H, Hentati B, AmmarKeskes L. Lipid peroxidation and antioxidant enzyme activities in infertile men: correlation with semen parameter. J Clin Lab Anal 2009; 23: 99-104.

29. Benedetti S, Tagliamonte MC, Catalani S, Primiterra M, Canestrari F, De Stefani S, et al. Differences in blood and semen oxidative status in fertile and infertile men, and their relationship with sperm quality. Reprod Biomed Online 2012; 25: 300-6.

30. Palmer NO, Bakos HW, Owens JA, Setchell BP, Lane M. Diet and exercise in an obese mouse fed a high-fat diet improve metabolic health and reverse perturbed sperm function. Am J Physiol Endocrinol Metab 2012; 302: 768-80. 